\title{
Clima Organizacional y desempeño laboral docente en la Asociación Educativa Adventista de la Misión Peruana del Sur, 2018.
}

\author{
Organizational Climate and teaching work performance in the Adventist Educational \\ Association of the Peruvian Mission of the South, 2018.
}

\author{
*Mamani Limache Diego Armando, Rivera Briceño Dannyd Moises, Marcos \\ Enrique Flores González. \\ a EP.Administración, Facultad de Ciencia Empresariales, Universidad Peruana Unión
}

INFORMACIÓN DEL ARTÍCULO

Historia del artículo

Recibido: agosto de 2018

Aceptado: setiembre de 2018

Palabras clave:

Desempeño laboral docente, clima organizacional

\section{Resumen}

El objetivo de la presente investigación fue determinar en qué medida se relaciona la realización personal y el desempeño laboral docente en la Asociación Educativa Adventista de la Misión Peruana del Sur, 2018. El estudio es de enfoque cuantitativo, de alcance correlacional, de diseño no experimental y de corte transversal. En el estudio participaron 120 docentes de la Asociación Educativa Adventista de la Misión Peruana del Sur, seleccionados de manera no probabilística. Para medir el clima organizacional, se aplicó el instrumento desarrollado y aplicado por Sonia Palma (2010), el Alfa de Cronbach en la prueba piloto fue de 0.978, con cinco dimensiones (realización personal, involucramiento laboral, supervisión, comunicación y condiciones laborales), y 11 indicadores. Para medir el desempeño laboral docente, se aplicó el instrumento desarrollado por Hernández (2010), el Alfa de Cronbach en la prueba piloto fue de 0.974 , con cuatro dimensiones (calidad de servicio, productividad, toma de decisiones y conocimiento), y 39 indicadores. Los resultados obtenidos mediante el Rho de Spearman es de 0.676 indicando que existe una relación directa, positiva y moderada, también se halló el $p$ valor que es igual a $0.000(p<0.05)$, lo cual significa que es altamente significativa. Se concluye, que el clima organizacional se relaciona de manera directa al desempeño laboral docente, y que a mayor comportamiento organizacional, mayor será el desempeño laboral o de lo contrario, a menor clima organizacional menor será el desempeño laboral. Al ser significativos estos resultados se pueden generalizar a poblaciones similares.

\section{Abstract}

The objective of this research was to determine the extent to which personal achievement and teacher work performance are related in the Adventist Educational Association of the Peruvian Mission of the South, 2018. The study is quantitative, correlational in scope, non-experimental design and cross section. The study involved 120 teachers from the Adventist Educational Association of the Peruvian Mission of the South, selected in a non-probabilistic manner. To measure the organizational climate, the instrument developed and applied by Sonia Palma (2010) was applied, Cronbach's Alpha in the pilot test was 0.978 , with five dimensions (personal fulfillment, work involvement, supervision, communication and working conditions), and 11 indicators. To measure teacher work performance, the instrument developed by Hernández (2010) was applied, Cronbach's Alpha in the pilot test was 0.974, with four 
Keywords:

Teacher work performance, organizational climate dimensions (quality of service, productivity, decision making and knowledge), and 39 indicators. The results obtained through Spearman's Rho is 0.676 , indicating that there is a direct, positive and moderate relationship, the $p$ value that is equal to 0.000 ( $p$ $<0.05$ ) was also found, which means that it is highly significant. It is concluded that the organizational climate is directly related to the teaching work performance, and that the greater the organizational behavior, the greater the work performance or otherwise, the lower the organizational climate, the lower the work performance. Being significant, these results can be generalized to similar populations.

\section{Introducción}

La globalización, los avances tecnológicos y las innovaciones, que se han dado en los últimos años han marcado los lineamientos de competencia, de calidad así como de la competitividad en las organizaciones, tales cambios disruptivos han obligado a las organizaciones a entrar en un proceso de renovación; obviamente en aquellas organizaciones que estuvieron preparadas, los cambios se convirtieron en oportunidades para poder consolidar su posicionamiento en el mercado que hoy por hoy es altamente competitivo; del mismo modo las organizaciones que no estuvieron preparadas y no pudieron salir airosas de este proceso quedaron rezagadas, desaparecieron o fueron absorbidas por la competencia.

En este contexto, se describe de manera panorámica, y surgen las siguientes interrogantes: ¿Qué debe mejorar una organización para ser competente y competitiva? ¿Su sistema de producción? ¿Sus estrategias administrativas? Si es en su sistema de producción, tendría que adquirir tecnología y así automatizar gran parte de sus procesos, y así poder mejorar la calidad del producto o servicio, como también para aumentar su capacidad, a fin de incrementar la oferta.

En este caso particular, la tecnología y la información están disponibles; se re- quiere entonces que la organización tenga la capacidad de inversión y de decisión, y así habría dado el primer paso en el proceso de transformación y de alineamiento con las exigencias del mercado. En cuanto al cambio de sus estrategias de administración, se avizora que allí radica la necesidad de una respuesta a esta situación problemática, para ello, la organización deberá imprimir su sello, estructurando su identidad su ADN, que lo caracterice y a la vez lo diferencie de las demás organizaciones e inclusive de las organizaciones del mismo rubro.

En pleno siglo XXI existen algunas organizaciones que aún conservan la herencia de una administración tradicional en en las cuales se percibe una brecha marcada a propósito de la comunicación entre el gerente y el colaborador, a propósito de los objetivos organizacionales se quedan en el nivel estratégico, como un privilegio de los altos mandos, esto es debido a que tales organizaciones centran su accionar en la parte operativa y en cómo satisfacer a los clientes externos, descuidando al cliente interno, cuyo rol protagónico debe ser reconocido como el más importante, ya que es quien ejecuta y realiza las actividades u operaciones para que la organización cumpla con su objetivo con eficacia, eficiencia y calidad. Es por esto que, la organización debe desarrolla planes estratégicos que consideren de manera prioritaria al individuo como eje fundamental 
del progreso, desarrollo y expansión organizacional.

Al respecto Regalado, Allpacca, Baca, \& Gerónimo (2011), a través del endomarketing ayudan a entender cómo debe ser la relación personal-organización, la cual tiene como propósito lograr que el personal de manera voluntaria oriente su esfuerzo para lograr la calidad del servicio, situación necesaria para lograr altos, consistentes y estables niveles de exigencia requeridos actualmente, tanto por los clientes internos como por los clientes externos, en todos los servicios que la organización pone a disposición.

Bajo el contexto de mantener satisfechos a los clientes internos y externos. Emergen la siguiente incógnita: ¿Cuál es el área de la organización encargada de promover la satisfacción del cliente interno y a través de qué? Según, Lumbreras (2017), en un estudio hecho, acerca de las principales gerencias que existen en una organización, a fin, de conocer cómo se distribuye el poder en la organización; de 1780 organizaciones el $77 \%$ mencionó a la Gerencia de Recursos Humanos como un área clave. La mayoría de estas organizaciones figuran en los primeros lugares del ranking especializado elaborado por Great Place to Work.

Indudablemente, diversos son los factores que deben considerarse en la gestión del talento humano, tal como: organización, remuneración, capacitación, evaluación del desempeño, evaluación de competencias, motivación y productividad.

A esta relación deben añadirse los deberes y los derechos tácitos de la organización. El conjunto de todos estos elementos y la manera como se desarrollen va a determinar el grado de relación entre la organización y el recurso humano, lo cual dependerá de cómo la organización establezca las prioridades entre los elementos $y$, a la vez, de si los elementos priorizados están en consonancia con las prioridades y las posibilidades del colaborador.

El clima laboral diferencia a las organizaciones exitosas de las mediocres. El ser humano es el eje principal de todo centro del trabajo, es el elemento más importante en la organización, y mientras este hecho no se asuma como tal, de nada vale hablar de sofisticadas herramientas de gestión.

A la luz de lo que dijeran Domínguez, Ramírez, \& García (2013), el clima laboral se forma a través de las percepciones personales, de igual forma abarca al conjunto de patrones recurrentes de conducta, actitudes y sentimientos que determinan el ambiente en una organización.

Por otro lado Olaz (2009), en un intento de definir el clima laboral, lo conceptualiza como un conjunto de variables situacionales de distinto orden y naturaleza, que oscilan en el transcurso del tiempo, afectando de desigual manera a todos aquellos miembros de la comunidad laboral.

Además, Chiavenato (2007), expresa que las acciones internas y externas (motivación), determinan en gran parte el accionar de los colaboradores. La manera en cómo asimile la motivación el colaborador exteriorizará su accionar positivo o negativo, para eso el colaborador debe de adaptarse a la situación y circunstancias, logrando un equilibrio emocional, operacionalizado en: sentirse bien consigo mismo, sentirse bien en su relación con otras personas y ser capaz de enfrentar 
las demandas de la vida. Esto es el resumen de clima organizacional, que está íntimamente relacionado con el grado de motivación de sus integrantes.

Asimismo, Fischman (2017), muestra los resultados de las investigaciones, en lo que se refiere a la evaluación del desempeño laboral. $50 \%$ de los empleados encuestados opinan que su evaluación de desempeño es injusta. En la cual, uno de cada cuatro empleados detesta las evaluaciones de desempeño laboral más que cualquier cosa en su vida laboral. Un $28 \%$ de los gerentes de recursos humanos piensa que las evaluaciones de desempeño son puro formalismo. Por otro lado, un estudio realizado por People IQ, revela que un $87 \%$ de los empleados y jefes encuestados, piensan que la evaluación del desempeño es improductiva.

Sin duda alguna, las organizaciones, deben encontrar estrategias en pro de difundir los beneficios que trae a la misma realizar las constantes evaluaciones, haciendo hincapié en el beneficio mutuo que generará el sincerar el rendimiento de cada colaborador, y como consecuencia, se pueda establecer una meritocracia para ascensos y aumentos de sueldo.

En este mismo orden de ideas, Álvarez, Blanca, Indacochea, Blanca, Álvarez, Arturo, Yoza, Narciso \& Figueroa (2018), aseveran que la evaluación de desempeño laboral es una herramienta indispensable en la gestión de las organizaciones, a fin de potenciar el talento humano, a través de una mejor calidad de vida, de tal manera que, una vez satisfechas sus necesidades pueda retribuir el interés de la organización, optimizando su productividad.

Al respecto, Goleman (1999), enfatiza que cada vez son más las organizaciones que alientan y propician la correcta evaluación del desempeño laboral debido a que, es un componente vital para la filosofía de la gerencia, pues actualmente no se compite sólo con productos, sino con la eficiente administración del talento humano.

Los resultados obtenidos en la evaluación del desempeño laboral, sirve a la gerencia para tomar las decisiones pertinentes a fin de fortalecer o corregir las habilidades y competencias de su recurso humano.

De modo que, la evaluación del desempeño laboral, beneficia de manera directa tanto al propio colaborador como a la misma organización. Estableciendo oportunidades para el trabajador dentro de ésta, mediante la promoción, ubicación en un puesto más acorde a sus habilidades, capacitación, aumento de sueldo, categorización, así como también en la asunción de responsabilidades de mando medio.

En palabras más sencillas, a la organización le permitiría categorizar sus colaboradores, establecer estándares y métricas de producción, optimizar los niveles de desempeño, reconocer el desempeño sobresaliente y lograr el compromiso e identificación de los mismos.

Ahora bien, en la Asociación Educativa Adventista de la Misión Peruana del Sur, se percibe una falta de involucramiento del personal docente con los propósitos de la organización, debido a que piensan que no pueden realizarse de manera personal ni profesional; además no sienten el apoyo ni la supervisión de los superiores; la comunicación no es fluida y consideran que no es específica, debido a que no se tiene un manual de funciones establecido; 
piensan finalmente que las condiciones laborales no son las óptimas. Agrupadas todas estas percepciones se tiene que el clima laboral no es el óptimo.

Por lo expuesto, se hizo pertinente llevar a cabo la presente investigación, a fin, de determinar en qué medida se relaciona el clima laboral y el desempeño laboral docente en la Asociación Educativa Adventista de la Misión Peruana del Sur, 2018.

\section{Clima organizacional}

El clima organizacional puede ser conceptualizado como un conjunto de características que describen a una organización, como una síntesis de percepciones del individuo y como un resultado del proceso de interacción social.

Para Méndez (2006), la conceptualización del clima organizacional parte de una interacción de las personas en un marco de una acción social que permite construir percepciones que por su origen e impacto colectivo se constituyen en atributos de la organización como un todo.

Además menciona que las conductas y patrones del comportamiento de los individuos en la organización son el resultado tanto del conjunto de valores, normas y pautas propias de la estructura organizacional, como de las condiciones que se establecen por el proceso de interacción; de tal manera que las experiencias vivenciales que cada individuo tiene en su entorno laboral son los que determinan las percepciones respecto de la organización (García \& Bejarano 2017).

Finalmente, todo lo mencionado anteriormente no tendría sentido sin que las características las cuales distinguen a una organización de otra y que perduran a tra- vés del tiempo influyan en el comportamiento de las personas formando una personalidad (Chiang, Heredia, \& Santamaría (2017).

\subsection{Tipos de clima organizacional}

Brunet (2011), citado por Ramos Moreno (2012), Likert aporta una serie de variables que se dan en la percepción del clima organizacional tales como estructura de la organización y de administración y dentro de ellas las reglas y normas, la toma de decisiones, motivaciones, las actitudes, la comunicación; también se incluyen la productividad, las ganancias y las pérdidas logradas en la organización.

Estos tipos de variables influyen en la percepción del clima por parte de los miembros de una organización, formándose un tipo de clima organizacional representativo en cada organización. A partir de diferentes configuraciones de variables, Likert llega a tipificar cuatro tipos de sistemas organizacionales, cada uno de ellos con un clima particular.

Ahora bien, el primero es el clima de tipo autoritario-explotador en este tipo de clima no existe la confianza entre los niveles de mando, tiene una naturaleza estrictamente autocrática.

En consecuencia, el miedo, temor, actitudes hostiles y la insatisfacción son fuerzas que predominan en los trabajadores. Además, las decisiones se toman en la alta dirección, que a su vez son poco motivantes y generalmente las toma un solo hombre.

Como segunda dimensión se encuentra el tipo autoritario-autoritarismo paternalista en la cual sigue prevaleciendo la desconfianza entre los niveles jerárquicos, sigue existiendo una comunicación 
deficiente, no obstante, se logra dar la comunicación, aunque ya se acotó en líneas previas es deficiente. Además, unas de las formas de motivación resultan ser los castigos.

En función a la tercera dimensión se presenta el tipo participativo-consultivo en el cual surge un cambio porque se empiezan a delegar algunas decisiones, es decir, algunas áreas son autónomas en cuanto a sus decisiones.

Además, las recompensas, los castigos ocasionales y cualquier implicación se utilizan para motivar a los trabajadores. Las actitudes son generalmente favorables y la mayor parte de los empleados se sienten responsables de lo que hacen.

Finalmente, y como cuarta dimensión está el tipo participativo-participación en grupo en el cual el clima es totalmente distinto al primero, porque se tiene una absoluta confianza. Los procesos de toma de decisiones están dirigidos a toda la organización, además la comunicación no solamente se da de manera vertical, sino que también se da de manera horizontal.

Entonces, los trabajadores están totalmente motivados por la participación y la consideración para el establecimiento de objetivos que dicho sea de paso se da en grupos en función del mejoramiento de métodos de trabajo.

\section{Desempeño laboral}

Sánchez Henríquez \& Calderón (2011), mencionan que a través del tiempo, las organizaciones han tomado mayor consideración y fuerza en la idea clave que determina el éxito a la forma en la que aprovechan el trabajo de sus empleados, siendo este un recurso muy valioso.
Debido a esa idea surgió el término "recursos humanos", naciendo también la necesidad de saber cómo están siendo administrados y si estos aportan a la organización con base en los parámetros que desea y que esta necesita.

Mientras que (Jesús \& Asch, 2015), asevera que en tiempos recientes y a través de la historia de la gestión del talento humano, siempre ha existido un debate continuo sobre cómo medir los recursos humanos de una manera precisa y confiable, llegando a optimizarlo para beneficio de la organización.

Ahora bien, en palabras de Pedraza, Amaya, \& Conde (2010), los cambios que se han generado en diferentes entornos laborales desde la antigüedad a esta parte han surtido efecto en pro de que las organizaciones aceleren su ritmo de trabajo para lograr buena competitividad y sean organizaciones exitosas.

Una de las cuales ha sido la evaluación del desempeño laboral de los empleados quienes residen en la organización; esto quiere decir que la organizaciones se ven obligados a diseñar y a aplicar sistemas de evaluación del desempeño para poder identificar de manera oportuna y eficaz fallas y limitaciones que no permitan el objeto de la organización

Asimismo, Chiavenato (2000), citado por (Quintero, Africano, \& Faría, 2008), enuncia sobre el comportamiento del trabajador en busca de los objetivos fijados, es decir la estrategia individual para lograr los objetivos. Entonces se afirma que un buen desempeño es la fortaleza más relevante con la que cuenta una organización.

De igual forma, Montejo (2001), define el desempeño como: "aquellas acciones o 
comportamientos observados en los empleados que son relevantes para los objetivos de la organización".

Finalmente, sobre la base de los aportado por Stone (2004) citado por Ruiz, Silva, \& Vena (2008), se tiene que el desempeño laboral es la manera cómo los individuos de la organización trabajan eficazmente, para alcanzar metas comunes, sujetos a las reglas básicas establecidas con anterioridad.

Igualmente, Chiavenato (1998), le define como: una sistemática apreciación del desempeño, del potencial de desarrollo del individuo en el cargo, afirmando que toda evaluación es un proceso para estimular o juzgar el valor, la excelencia, las cualidades de alguna persona.

\subsection{Teorías de desempeño laboral}

Según Olivera (2013), la motivación es producto de tres conceptos básicos.

Valencia: Valor que para la persona tiene el resultado de su acción.

Instrumental: siendo la anticipación subjetiva sobre la probabilidad de que un resultado, le sirve para obtener otros resultados. Y por último.

Expectativa: Siendo la probabilidad de que cierto esfuerzo conduzca a cierto resultado de un primer nivel, es decir, la estimulación que se tiene en función de que la probabilidad de que el esfuerzo produzca un desempeño óptimo es alta.

Basado en las consideraciones anteriores, un empleado tendrá buen rendimiento si llega a la siguiente conclusión: su esfuerzo influye en el rendimiento laboral y este en otros resultados positivos y atractivos para él; siendo este su rendimiento.
En cambio; disminuirá si llega a la conclusión de que estos resultados no dependen de su esfuerzo. Los empleados se sentirán motivados para aumentar su desempeño, si estos consideran que ello traerá como consecuencia una buena evaluación del mismo.

\subsubsection{Teoría de la equidad}

Hernan \& Alviter (2006), muestran ante la comunidad científica la teoría de la equidad de Adams (1965), los cuales proponen que los individuos están motivados a ser tratados equitativamente en relación con su desempeño y el desempeño de sus compañeros.

Por otro lado Olivera (2013), menciona que el factor primordial para la motivación en el trabajo, es la evaluación individual en cuanto a la equidad y la justicia de la recompensa recibida.

Entonces un término de equidad se define como una parte que guarda los insumos laborales del individuo y las recompensas laborales. Entonces según esta teoría los individuos están motivados cuando experimentan satisfacción con lo que reciben de acuerdo con el esfuerzo hecho.

Por último, Vega (2007), citado por Chávez \& Puyo Huayunga (2017), mencionan que la equidad es el punto medio entre las cualidades laborales del trabajador y las recompensas que reciben a cambio.

Esto quiere decir que de acuerdo con la teoría, los trabajadores se motivan cuando llegan a experimentar satisfacción por lo recibido mediante el esfuerzo realizado. 
3.2.1. Teoría de Establecimiento de Metas y Retroalimentación

Molina (2012), Menciona que los empleados necesitan saber cómo van en la medida en la que avanzan hacia el logro de sus metas. Las fuentes de retroalimentación pueden venir de la tarea misma, pero en ciertos casos proceden de un supervisor.

Según Betancur (2010), citado por; Chávez \& Puyo Huayunga (2017), el trabajador debe poseer posibilidades de saber cómo progresa en su camino hacia el cumplimiento de las metas. Sabiendo que no es suficiente establecer metas específicas y con la retroalimentación, existen otros factores que influyen sobre el desempeño como el compromiso por parte del trabajador.

En definitiva, esta teoría también sostiene que las metas tanto específicas como metas difíciles son las que llevan a un alto desempeño laboral.

\section{Materiales y métodos}

La investigación realizada en función de escribir este artículo científico es de tipo cuantitativo, descriptiva y correlacional.

Es cuantitativa, en tanto que los datos recolectados serán procesados, cuantificados y procesados, haciendo uso de la matemática y la estadística; es descriptiva, porque a través de la percepción y la observación se puntualiza el comportamiento organizacional, sin influir de manera directa o indirecta en los individuos participantes de la misma; es correlacional porque pretende establecer en qué medida se relacionan las variables: trabajo en equipo y desempeño docente.
El estudio es de diseño no experimental de corte transversal; no experimental, debido a que no se manipulan las variables y transversal, en cuanto a que la información recolectada es en un tiempo determinado y por única vez.

\subsection{Participantes}

En palabras de Arias (2006), una población es el conjunto de todos los elementos con características comunes y puede ser de carácter finito o infinito, dependiendo de las limitaciones expuestas en el problema y por los objetivos planteados de estudio. Las conclusiones de la investigación permitieron inferir sobre el comportamiento de la población.

Para el trabajo de investigación la población objeto de estudio, está formada por los docentes, de la Asociación Educativa Adventista de la Misión Peruana del Sur, en sus tres niveles: inicial, primaria y secundaria, siendo un total de 120 docentes.

La muestra incluyó la incorporación de todos los docentes, salvo aquellos que estuvieron de licencia o se negaron a colaborar.

\subsection{Instrumentos}

El instrumento que permitió medir la variable independiente es la escala de Clima Laboral de Sonia Palma Carrillo, la cual describe el nivel de percepción global del ambiente laboral y específico en relación con la realización personal, involucramiento laboral, supervisión y condiciones laborales.

Ahora bien, la calificación del instrumento de acuerdo con las normas técnicas establecidas se puntúa de 1 a 5 , con opciones de respuesta en función con la 
escala de Likert ( $1=$ Totalmente en desacuerdo, 2=En desacuerdo, 3=Ni en acuerdo ni en desacuerdo, 4=De acuerdo y $5=$ Totalmente en acuerdo), y con un total de 250 puntos como máximo. Las categorías diagnósticas consideradas para el instrumento, están basadas en las puntuaciones directas del instrumento y tomando como criterio que la máxima puntuación, revela una mejor percepción del ambiente de trabajo y viceversa.

Por otro lado, el instrumento que permitirá medir la variable dependiente es la escala de Desempeño Laboral de Hernández, la cual describe el nivel desempeño laboral en relación con la calidad de servicio, productividad, toma de decisiones y el conocimiento.

En suma, la calificación del instrumento de acuerdo con las normas técnicas establecidas también se puntúa de 1 a 5 puntos, también con opciones de respuesta en función con la escala de Likert (1=Totalmente en desacuerdo, 2 = En desacu- erdo, 3=Ni en acuerdo ni en desacuerdo, 4=De acuerdo y 5=Totalmente en acuerdo) y con un total de 195 puntos como máximo.

Las categorías diagnósticas consideradas para el instrumento están basadas en las puntuaciones directas del instrumento y tomando como criterio que la máxima puntuación, revela una mejor percepción del desempeño y viceversa.

En la Tabla 1, se presenta el análisis de confiabilidad mediante el Alfa de Cronbach, el cual para el instrumento de la variable clima organizacional, tiene un coeficiente de 0.978 el cual cuenta con 50 indicadores, y el instrumento de la variable desempeño laboral contentivo de un coeficiente de 0.974 , que contiene 39 indicadores.

En conclusión, ambos instrumentos tienen alta consistencia interna y alto nivel de confiabilidad, por lo tanto, se puede aplicar en poblaciones similares.

Tabla 1

Confiabilidad del instrumento

\begin{tabular}{lcc}
\hline Variables & Alfa de Cronbach & N de Ítems \\
\hline Clima organizacional & 0.978 & 50 \\
Desempeño laboral & 0.974 & 39 \\
\hline
\end{tabular}

\subsection{Análisis de datos}

Se solicitó una carta de presentación por parte de la Dirección de la Escuela Profesional de Administración, en la cual se evidenciaba el respaldo de la Institución hacia los investigadores; con tal documento se procedió a requerir el permiso correspondiente a la los administradores de la Misión Peruana del Sur, como promotora de la Asociación Educativa Adventista en esa región.
En definitiva, para el procesamiento de la información se construyó una matriz de datos usando el programa informático Microsoft Excel, en función de identificar y registrar los datos correspondientes a cada ítem formulado en los cuestionarios, los cuales midieron a cada variable especificada (clima organizacional y desempeño laboral), para luego realizar la exportación de datos al programa estadístico SPSS 22.0 en donde, a través de las diferentes opciones y bondades del programa 
se obtuvieron los reportes, que permitieron hacer el análisis, evaluación y discusión de los datos.

\section{Resultados y Discusión}

\subsection{Resultados sociodemográficos}

De los datos presentados en la Tabla 2 , se tiene como conclusión que en la red educativa adventista de la Misión Peruana del Sur el $65 \%$ del personal docente es fe- menino, que guarda relación con los datos presentados por INEI (2018), en donde del total de docentes en todo el Perú el $63.6 \%$ son mujeres.

Además, que el $57.5 \%$ son casados y tienen una experiencia mayor a 11 años con un $43.3 \%$. Por el nivel de enseñanza el $43.3 \%$ es del nivel primario, que permite intuir su potencial fortalecido en la educación básica.

Tabla 2

Información sociodemográfica de los participantes

\begin{tabular}{|c|c|c|c|}
\hline & & Frecuencia & Porcentaje \\
\hline \multirow{3}{*}{ Género } & Masculino Femenino & 41 & $34.2 \%$ \\
\hline & & 79 & $65.8 \%$ \\
\hline & Total & 120 & $100.0 \%$ \\
\hline \multirow{5}{*}{ Estado Civil } & Soltero & 46 & $38.3 \%$ \\
\hline & Casado & 69 & $57.5 \%$ \\
\hline & Viudo & 0 & $0.0 \%$ \\
\hline & Divorciado & 5 & $4.2 \%$ \\
\hline & Total & 120 & $100.0 \%$ \\
\hline \multirow{9}{*}{ Institución Educativa } & San Martin & 7 & $5.8 \%$ \\
\hline & Fernando Forga & 1415 & $11.7 \% 12.5 \%$ \\
\hline & Fernando Sthal & 30 & $25.0 \% 24.2 \%$ \\
\hline & 28 de Julio El & 29 & $5.8 \%$ \\
\hline & Faro & 7 & \\
\hline & Mirave & & \\
\hline & Maranatha & 7 & $5.8 \%$ \\
\hline & Majes & 11 & $9.2 \%$ \\
\hline & Total & 120 & $100.0 \%$ \\
\hline \multirow{4}{*}{ Nivel de Enseñanza } & Inicial & 25 & $20.8 \% 43.3 \%$ \\
\hline & Primaria & 52 & $35.8 \%$ \\
\hline & Secundaria & 43 & \\
\hline & Total & 120 & $100.0 \%$ \\
\hline
\end{tabular}




\begin{tabular}{lccc}
\hline & Misionero Empleado & 0 & $0.0 \%$ \\
Condición Laboral & & 55 & $45.8 \%$ \\
& Contratado & 65 & $54.2 \%$ \\
& Total & 120 & $100.0 \%$ \\
\hline \multirow{2}{*}{ Experiencia Profesional } & $1-5$ años & 36 & $30.0 \% 26.7 \%$ \\
& $6-10$ años & 32 & $25.8 \%$ \\
& $11-15$ años & 31 & $10.8 \%$ \\
& $16-20$ años & 13 & $6.7 \%$ \\
& 21 a más años & 8 & $100.0 \%$ \\
\hline
\end{tabular}

5.2. Resultados de comprobación de hipótesis

\subsubsection{Hipótesis general}

Existe relación estadísticamente significativa entre clima organizacional $y$ desempeño laboral docente en la Asociación Educativa Adventista de la Misión Peruana del Sur, 2018

Ho: No existe relación significativa entre clima organizacional y desempeño laboral docente en la Asociación Educativa Adventista de la Misión Peruana del Sur, 2018.

Ha: Existe relación significativa entre clima organizacional y desempeño laboral docente en la Asociación Educativa Ad- ventista de la Misión Peruana del Sur, 2018,

Se observa en la Tabla 3 la relación existente entre clima organizacional y desempeño laboral, revelándose que la relación es directa y moderada de acuerdo con lo que indica el estadístico de correlación Rho de Spearman (rho $=.676^{* *}$ ), de igual modo muestra que el nivel de significancia o $p$ valor es igual a 0.000 siendo menor a 0.05 , lo que indica que la relación entre ambas variables es altamente significativa, de tal modo se rechaza la hipótesis nula y se acepta la alterna. Se concluye que, a mayor comportamiento organizacional, mayor será el desempeño laboral o de lo contrario, a menor comportamiento organizacional menor será el desempeño laboral.

Tabla 3

Relación entre clima organizacional y desempeño laboral docente

\begin{tabular}{lccc} 
& \multicolumn{1}{c}{ Desempeño laboral } & & \\
& Rho de Spearman & Sig. (bilateral) & $\mathrm{N}$ \\
\hline Comportamiento organizacional &, $676^{* *}$ & .000 & 120 \\
\hline$* *$ la correlación es significativa en el nivel 0,01 (bilateral)
\end{tabular}

**. La correlación es significativa en el nivel 0,01 (bilateral). 


\subsubsection{Hipótesis especifica 1}

Ho: No existe relación significativa entre la realización personal y el desempeño laboral docente en la Asociación Educativa Adventista de la Misión Peruana del Sur, 2018.

Ha: Existe relación significativa entre la realización personal y el desempeño laboral docente en la Asociación Educativa Adventista de la Misión Peruana del Sur, 2018.

Se observa en la Tabla 4 la relación existente entre realización personal y de- sempeño laboral docente, se aprecia que la relación es directa y moderada de acuerdo con lo que indica el estadístico de correlación Rho de Spearman $\left(\right.$ rho $=.557^{* *}$ ), de igual modo muestra que el nivel de significancia o $p$ valor es igual a 0.000 siendo menor a 0.05 , lo que indica que la relación entre ambas variables es altamente significativa, de tal modo se rechaza la hipótesis nula y se acepta la alterna. Se concluye que, a mayor nivel de realización personal, mayor será el desempeño laboral o de lo contrario, a menor nivel de realización personal menor será el desempeño laboral.

Tabla 4

Relación entre realización personal y desempeño laboral docente

\begin{tabular}{|c|c|c|c|}
\hline & mpeño laboral & & \\
\hline & Rho de Spearman & Sig. (bilateral) & $\mathrm{N}$ \\
\hline Realización personal &, $557 * *$ & .000 & 120 \\
\hline
\end{tabular}

\subsubsection{Hipótesis especifica 2}

Ho: No existe relación significativa entre el involucramiento laboral y el desempeño laboral docente en la Asociación Educativa Adventista de la Misión Peruana del Sur, 2018.

Ha: Existe relación significativa entre el involucramiento laboral y el desempeño laboral docente en la Asociación Educativa Adventista de la Misión Peruana del Sur, 2018.

Se observa en la Tabla 5 la relación existente entre involucramiento laboral y desempeño laboral docente, se aprecia que la relación es directa y moderada de acuerdo con lo que indica el estadístico de correlación Rho de Spearman (rho $=.574^{* *}$ ), de igual modo muestra que el nivel de significancia o $p$ valor es igual a 0.000 siendo menor a 0.05 , indicando que la relación entre ambas variables es altamente significativa, de tal modo se rechaza la hipótesis nula y se acepta la alterna.

En conclusión, a mayor nivel de realización personal, mayor será el desempeño laboral o de lo contrario, a menor nivel de realización personal menor será el desempeño laboral. 
Tabla 5

Relación entre desempeño laboral e involucramiento laboral docente

\begin{tabular}{lccc}
\hline \multicolumn{3}{c}{ Desempeño laboral } & \\
& Rho de Spearman & Sig. (bilateral) & $\mathrm{N}$ \\
\hline Involucramiento laboral &, $574^{* *}$ & .000 & 120 \\
\hline${ }^{* *}$. La correlación es significativa en el nivel 0,01 (bilateral). & &
\end{tabular}

\subsubsection{Hipótesis especifica 3}

Ho: No existe relación significativa entre las condiciones laborales y el desempeño laboral docente en la Asociación Educativa Adventista de la Misión Peruana del Sur, 2018.

Ha: Existe relación significativa entre las condiciones laborales y el desempeño laboral docente en la Asociación Educativa Adventista de la Misión Peruana del Sur, 2018.

Se observa en la Tabla 6 la relación existente entre condiciones laborales y desempeño laboral docente, revela que la relación es directa y moderada de acuerdo con lo que indica el estadístico de correlación Rho de Spearman $\left(\right.$ rho $=.651^{* *}$ ), de igual modo muestra que el nivel de significancia o $p$ valor es igual a 0.000 siendo menor a 0.05 , lo que indica que la relación entre ambas variables es altamente significativa, de tal modo se rechaza la hipótesis nula y se acepta la alterna. Se concluye que, a mayor nivel de las condiciones laborales, mayor será el desempeño laboral docente o de lo contrario, a menor nivel en las condiciones laborales menor será el desempeño laboral docente.

Tabla 6

Relación entre el trabajo en equipo y la evaluación docente.

\begin{tabular}{lccc}
\hline \multicolumn{3}{c}{ Desempeño laboral } & \\
& Rho de Spearman & Sig. (bilateral) & $\mathrm{N}$ \\
\hline Condiciones laborales &, $651^{* *}$ & .000 & 120 \\
\hline${ }^{* *}$. La correlación es significativa en el nivel 0,01 (bilateral). & &
\end{tabular}

\subsubsection{Hipótesis especifica 4}

Ho: No existe relación significativa entre la comunicación y el desempeño laboral docente en la Asociación Educativa Adventista de la Misión Peruana del Sur, 2018.

Ha: Existe relación significativa entre la comunicación y el desempeño laboral docente en la Asociación Educativa Adventista de la Misión Peruana del Sur, 2018.
Se observa en la Tabla 7 la relación existente entre comunicación y desempeño laboral docente, se aprecia que la relación es directa y moderada de acuerdo con lo que indica el estadístico de correlación Rho de Spearman (rho $=.650^{* *}$ ), de igual modo muestra que el nivel de significancia o $p$ valor es igual a 0.000 siendo menor a 0.05 , lo que indica que la relación entre ambas variables es altamente significativa, de tal modo se rechaza la hipótesis nula y se acepta la alterna. 
En definitiva, a mayor nivel de la comunicación, mayor será el desempeño laboral docente o de lo contrario, a menor nivel en la comunicación menor será el desempeño laboral docente.

Tabla 7

Relación entre comunicación y desempeño laboral docente

Desempeño laboral

\begin{tabular}{lccc}
\hline & Rho de Spearman & Sig. (bilateral) & N \\
\hline Comunicación &, $650^{* *}$ & .000 & 120
\end{tabular}

**. La correlación es significativa en el nivel 0,01 (bilateral).

\subsubsection{Hipótesis especifica 5}

Ho: No existe relación significativa entre la supervisión y el desempeño laboral docente en la Asociación Educativa Adventista de la Misión Peruana del Sur, 2018.

Ha: Existe relación significativa entre la supervisión y el desempeño laboral docente en la Asociación Educativa Adventista de la Misión Peruana del Sur, 2018.

Se observa en la Tabla 8 la relación existente entre supervisión y desempeño laboral docente, apreciándose que la rela- ción es directa y moderada de acuerdo con lo que indica el estadístico de correlación Rho de Spearman $\left(r h o=.669^{* *}\right)$, de igual modo revela que el nivel de significancia o $p$ valor es igual a 0.000 siendo menor a 0.05 , lo que indica que la relación entre ambas variables es altamente significativa, de tal modo se rechaza la hipótesis nula y se acepta la alterna.

En conclusión, a mayor nivel de la supervisión, mayor será el desempeño laboral docente o de lo contrario, a menor nivel en la supervisión menor será el desempeño laboral docente.

Tabla 8

Relación entre supervisión y desempeño laboral docente

\begin{tabular}{lccc}
\hline \multicolumn{3}{c}{ Desempeño laboral } & \\
& Rho de Spearman & Sig. (bilateral) & $\mathrm{N}$ \\
\hline Supervisión &, $669^{* *}$ & .000 & 120 \\
\hline${ }^{* *}$. La correlación es significativa en el nivel 0,01 (bilateral).
\end{tabular}




\section{Conclusiones}

Con base en los resultados obtenidos dentro del proceso investigativo vivido, las conclusiones quedan como sigue:

Primera: El clima organizacional guarda relación estadísticamente significativa y directa con el desempeño laboral docente de la Asociación Educativa Adventista de la Misión Peruana del Sur. Lo que significa que a mejor clima organizacional, mejor será el desempeño laboral docente. Con un estadístico de correlación Rho de Spearman $\left(\mathrm{rho}=.676^{* *}\right)$; y el valor $p=, 000$, visibilizando que la relación entre ambas variables es altamente significativa. Tomando en cuenta el compromiso con el éxito de la organización y un trabajo retador como pilares de influencia.

Segunda: La realización personal guarda relación estadísticamente significativa y directa con el desempeño laboral docente de la Asociación Educativa Adventista de la Misión Peruana del Sur. Lo que significa que a mejor realización personal, mejor será el desempeño laboral docente. Con un estadístico de correlación Rho de Spearman $\left(\right.$ rho $\left.=.557^{* \star}\right)$; y el valor $p=, 000$, evidenciando que la relación entre ambas variables es altamente significativa. Resaltando que el docente se siente factor clave para el éxito de la organización y participando en la definición de objetivos y acciones para lograrlo.

Tercera: El involucramiento laboral guarda relación estadísticamente significativa y directa con el desempeño laboral docente de la Asociación Educativa Adventista de la Misión Peruana del Sur. Lo que significa que a mejor involucramiento laboral, mejor será el desempeño laboral docente. Con un estadístico de correlación Rho de Spearman $\left(\right.$ rho $\left.=.574^{* *}\right)$; y el valor $p=, 000$, indicando que la relación entre ambas variables es altamente significativa. Resaltando que, el docente se siente un factor clave para el éxito de la institución percibiéndose a través de las evaluaciones que se hacen del mismo.

Cuarta: Las condiciones laborales guarda relación estadísticamente significativa y directa con el desempeño laboral docente de la Asociación Educativa Adventista de la Misión Peruana del Sur. Lo que significa que a mejor condiciones laborales, mejor será el desempeño laboral docente. Con un estadístico de correlación Rho de Spearman (rho $\left.=.651^{* *}\right)$; y el valor $p=, 000$, lo que indica que también la relación entre ambas variables es altamente significativa. Se asume que los docentes sienten que cumpliendo las tareas diarias en el trabajo se alcanza el desarrollo del personal superando y afrontando los obstáculos que se presenten.

Quinta: La comunicación guarda relación estadísticamente significativa y directa con el desempeño laboral docente de la Asociación Educativa Adventista de la Misión Peruana del Sur. Lo que significa que a mejor comunicación, mejor será el desempeño laboral docente. Con un estadístico de correlación Rho de Spearman (rho $=.650^{* *}$ ); y el valor $p=, 000$, el cual revela que la relación entre ambas variables es altamente significativa. Subrayando que los objetivos están claramente definidos de tal modo que todos hacen esfuerzos conjuntos y unánimes para alcanzar tales. A su vez, los resultados se usan como base favorable en las relaciones internas y externas.

Sexta: La supervisión guarda relación estadísticamente significativa y directa con el desempeño laboral docente de la Asociación Educativa Adventista de la Misión Peruana del Sur. Indicando que, a mejor supervisión, mejor será el desem- 
peño laboral docente. Con un estadístico de correlación Rho de Spearman (rho $\left.=.669^{* *}\right)$; y el valor $p=, 000$, indícalo que significa es que también la relación entre ambas variables es altamente significativa. Resaltando que se dispone de una tecnología que facilita el trabajo del docente y colaboración entre el personal; de tal modo que se podrá promover la generación de ideas creativas innovadoras.

\section{Recomendaciones}

Tomando en cuenta los resultados de esta investigación respecto al moderado clima organizacional y al moderado desempeño laboral se recomienda realizar un programa de apoyo organizacional con los administradores y tomadores de decisiones a fin de que esta pueda repercutir en una mejor percepción y sentir de los colaboradores en el clima organizacional.

Además de ello se sugiere al personal administrativo implementar un programa de crecimiento organizacional y/o estructural, con lo que el docente pueda aspirar y ejercer cargos administrativos. Deben organizar y planificar sesiones de capacitación al docente para que pueda estar a la vanguardia de la mejora educativa.

De la misma manera, se exhorta a la asociación realizar un plan de reconocimiento personal por periodo a fin de que el docente sepa que tiene otras oportunidades para mejorar y poder ser reconocido.

Por otro lado, se invita a mejorar en cierta medida estratégica la remuneración salarial de los docentes, respecto al trabajo natural que realizan. También la mejora de los incentivos acordes con el desempeño y logros en las instituciones.

Adicionalmente, recomienda, tener en consideración la cantidad de mujeres en la solución de conflictos y responsabilidad de tareas. Finalmente, es preciso otorgar estabilidad laboral a los docentes a los cuales por ley les corresponde. 


\section{Referencias}

Apolo, D., Báez, V., Pauker, L., \& Pasquel, G. (2017). Gestión de comunicación corporativa: consideraciones para el abordaje de su estudio y práctica. Revista Latina de Comunicación Social, (72), https://doi.org/10.4185/RLCS

Bardales, V. (2016). " Clima organizacional y desempeño laboral de los trabajadores de la Unidad de Gestión Educativa Local - Mariscal Cáceres - Juanjui . Año. Universidad César Vallejo.

Barre Aveiga, D. J. (2014). "Estudio sobre la satisfacción laboral y su relación con la rotación de personal de Auxiliares de Punto de Venta y de Servicios Generales de las Pharmacy's del Norte de la ciudad de Guayaquil, durante el periodo de Enero-Agosto 2013". Satisfacción Laboral.

Chávez, E., \& Puyo Huayunga, E. (2017). Relacion entre el Clima Organizacional y el desempeño laboral de los colaboradores del area administrativa en las empresas constructoras del distrito de tarapoto, 2017.

Retrieved from http://repositorio.upeu.edu.pe/bitstream/ handle/UPEU/896/Enrique_tesis_Bachill er_2017.pdf? sequence $=1 \overline{\text { \&isAllowed }}=\mathrm{y}$

Chiang Vega, M. M., Heredia Gálvez, S. A., \& Santamaría freire, E. J. (2017). Clima organizacional y salud psicológica: una dualidad organizacional. Dimensión Empresarial, 15(1), 63-76. Retrieved from

https://dialnet.unirioja.es/servlet/articulo ?codigo $=5826333$

Chiavenato, I. (2007). Administración de recursos humanos. McGraw-Hill / Interamericana. https://doi.org/10.1007/s13398-0140173-7.2
Fischman, D. (2017, May). "Evaluaciones de desempeño", por David Fischman. El Comercio, p. 1.

García, J., \& Bejarano, J. (2017). Relación entre el clima organizacional y satisfacción laboral de los colaboradores de la Asociación de Productores y Abastecedores de cereales $y$ oleaginosas de Los Ríos del cantón Mocache-Ecuador, 2016, 2(2), 97-108.

Goleman, D. (1999). La práctica de la inteligencia emocional. (Kairos SA, Ed.). Barcelona. España.

Hernan, F., \& Alviter, E. (2006). LA TEORÍA DE EQUIDAD DE ADAMS DESDE EL PUNTO DE VISTA DEL ABUSIVO. Retrieved from http://acacia.org.mx/busqueda/pdf/16_0 3_teoria_de_la_equidad.pdf

Herrera, Gleydi. (2016). La inteligencia emocional y su relacion con el desepeño laboral de los trabajadores de la municipalidad Provincial de San Martin, Region de San Martin, 2016. Retrieved from

http://repositorio.upeu.edu.pe/bitstream/ handle/UPEU/400/Gleydi Tesis bachill er_2017.pdf?s equence $=\overline{1}$ \&isAllowed $=y$

Jesús, I., \& Asch, M. (2015). La evaluación del desempeño en las empresas y la resiliencia: Una revisión de literatura Performance review in companies and resilience: A literature review. Retrieved from

https://ufidelitas.ac.cr/assets/es/revistafidelitas/se-2-ciencia-5-morganjesus.pdf

Lumbreras. (2017, May). ¿La rentabilidad de una empresa depende de su clima laboral? El Comercio, p. 1.

Molina, H. (2012). ESTABLECIMIENTO DE METAS, COMPORTAMIENTO Y DESEMPEÑO. Retrieved from 
http://www.scielo.org.co/pdf/eg/v16n75/v 16n75a02.pdf

Montejo, A. P. (2001). Evaluacion del Desempeño Laboral. Charles-Henri, (2), 222. Retrieved from http://www.monografias.com/cgi-bin/

Olaz, Á. J. (2009). Definición de un modelo de clima laboral basado en la gestión por competencias. Revista de Sociologia, (91), 193-201.

Olivera, Y. (2013). Estudio de la Motivacion y su influencia en el desempeño laboral de los Empleados Administrativos del Area Comercial de la Constructora Furoiani Obras y Proyectos. Retrieved from http://repositorio.ug.edu.ec/bitstream/red ug/10432/1/TESIS DE GRADO YOLANDA OLVERA.pdf

Pedraza, E. *, Amaya, G., \& Conde. (2010). Desempeño laboral y estabilidad del personal administrativo contratado de la Facultad de Medicina de la Universidad del Zulia. Revista de Ciencias Sociales (RCS), XVI(3), 493-505. Retrieved from

http://www.redalyc.org/pdf/280/2801632 0010.pdf

Quintero, N., Africano, N., \& Faría, E. (2008). CLIMA ORGANIZACIONAL Y DESEMPEÑO LABORAL DEL PERSONAL EMPRESA VIGILANTES ASOCIADOS COSTA ORIENTAL DEL LAGO ORGANIZATIONAL CLIMATE AND LABOUR PERFORMANCE OF
THE COMPANY \&quot;VIGILANTES ASSOCIATE\&quot; ORIENTAL COAST OF THE LAKE. Retrieved from http://ojs.revistanegotium.org.ve/index.p hp/negotium/article/viewFile/57/49

Regalado, O., Allpacca, R., Baca, L., \& Gerónimo, M. (2011). Endomárketing: estrategias de relación con el cliente interno. (U. ESAN, Ed.), Journal of Chemical Information and Modeling

(Primera ed, Vol. 53). Lima. Perú. https://doi.org/10.1017/CBO9781107415 324.004

Ruiz, J., Silva, N., \& Vena, M. (2008). Etica empresarial y el desempeño laboral en organizaciones de alta tecnologia. Revista Venezolana de Gerencia, 13(43). Retrieved from http://www.redalyc.org/html/290/290043 06/

Sánchez, D., \& Naranjo, Y. (2014). El proceso administrativo: Factor determinante en las Mipymes colombianas del siglo XXI. Retrieved from http://congreso.investiga.fca.unam.mx/d ocs/xix/docs/1.16.pdf

Sánchez Henríquez, J., \& Calderón, V. C. (2011). Diseño del proceso de evaluación del desempeño del personal y las principales tendencias que afectan su auditoría. Retrieved from http://www.redalyc.org/pdf/646/6462393 2004.pdf 